\title{
Cardiovascular Complications in Patients with COVID-19: Consequences of Viral Toxicities and Host Immune Response
}

\author{
Han Zhu ${ }^{1,2,3}$. June-Wha Rhee ${ }^{1,2,3} \cdot$ Paul Cheng ${ }^{1,2,3} \cdot$ Sarah Waliany ${ }^{1}$. Amy Chang ${ }^{1,4} \cdot$ Ronald M. Witteles ${ }^{1,3}$. \\ Holden Maecker $^{5,6} \cdot$ Mark M. Davis ${ }^{5,6,7} \cdot$ Patricia K. Nguyen ${ }^{1,2,3} \cdot$ Sean M. Wu ${ }^{1,2,3}$
}

Published online: 21 April 2020

(C) Springer Science+Business Media, LLC, part of Springer Nature 2020, corrected publication 2020

\begin{abstract}
Purpose of Review Coronavirus disease of 2019 (COVID-19) is a cause of significant morbidity and mortality worldwide. While cardiac injury has been demonstrated in critically ill COVID-19 patients, the mechanism of injury remains unclear. Here, we review our current knowledge of the biology of SARS-CoV-2 and the potential mechanisms of myocardial injury due to viral toxicities and host immune responses.

Recent Findings A number of studies have reported an epidemiological association between history of cardiac disease and worsened outcome during COVID infection. Development of new onset myocardial injury during COVID-19 also increases mortality. While limited data exist, potential mechanisms of cardiac injury include direct viral entry through the angiotensinconverting enzyme 2 (ACE2) receptor and toxicity in host cells, hypoxia-related myocyte injury, and immune-mediated cytokine release syndrome. Potential treatments for reducing viral infection and excessive immune responses are also discussed.

Summary COVID patients with cardiac disease history or acquire new cardiac injury are at an increased risk for in-hospital morbidity and mortality. More studies are needed to address the mechanism of cardiotoxicity and the treatments that can minimize permanent damage to the cardiovascular system.
\end{abstract}

Keywords COVID-19 · SARS-CoV-2 · Immune response $\cdot$ Cardiovascular system · Cardiac injury $\cdot$ Cytokine storm

Sean M. Wu

smwu@stanford.edu

Han Zhu

hanzhu@stanford.edu

June-Wha Rhee

jwrhee@stanford.edu

Paul Cheng

chengpa@stanford.edu

Sarah Waliany

swaliany@stanford.edu

Amy Chang

axc421@stanford.edu

Ronald M. Witteles

witteles@stanford.edu

Holden Maecker

maecker@stanford.edu
Mark M. Davis

mmdavis@stanford.edu

Patricia K. Nguyen

pknguyen@stanford.edu

1 Department of Medicine, Stanford University, Room G1120A, Lokey Stem Cell Building, 265 Campus Drive, Stanford, CA 94305, USA

2 Stanford Cardiovascular Institute, Stanford, CA, USA

3 Division of Cardiovascular Medicine, Stanford University, Stanford, CA, USA

4 Division of Infectious Disease, Stanford University, Stanford, CA, USA

5 Department of Microbiology and Immunology, Stanford University, Stanford, CA, USA

6 Stanford Institute for Immunity, Transplantation and Infection, Stanford University School of Medicine, Stanford, CA, USA

7 Howard Hughes Medical Institute, Stanford, CA, USA 


\section{Introduction}

Coronavirus disease of 2019 (COVID-19), caused by infection from severe acute respiratory syndrome coronavirus 2 (SARS-CoV-2), has spread across the world as a serious pandemic $[1,2]$. SARS-CoV-2, an enveloped virus with non-segmented, single-stranded, positive-sense RNA genome [3], is a member of the Coronaviridae (CoV) family which causes a predominantly respiratory illness with a wide range of clinical severity, ranging from asymptomatic or mildly symptomatic (fever, cough, dyspnea, myalgias, fatigue, and diarrhea) in a large proportion of patients to severe acute respiratory distress syndrome (ARDS) and fatal multi-organ failure $[1,4-6,7 \bullet \bullet]$. The disease has a case-fatality rate that ranges from less than $0.5 \%$ to more than $7 \%$ (average, $\sim 3.8 \%$ ) [8], with an infectivity greater than that of influenza [9]. Its high transmissibility and relatively high rate of causing serious complications has led COVID-19 to become a serious public health threat worldwide.

Among various physiological consequences of severe COVID-19, cardiovascular complications have emerged as some of the most significant and life threatening. COVID-19 may present with respiratory failure from pneumonia and ARDS, with or without distributive \pm cardiogenic shock [10, $11,12 \bullet]$, and severe cardiac injury manifesting as markedly elevated troponin and heart failure [12•, 13-14]. Cardiac injury has also been associated with increased mortality [15•0]. In a cohort study of 416 patients with confirmed COVID-19, elevated troponin was present in $19.7 \%$ of patients during hospitalization and was found to be an independent risk factor for in-hospital mortality [15••]. The increased incidence of cardiac injury among those with severe systemic inflammatory response syndromes (SIRS) and shock in the setting of COVID-19 also highlights an important relationship between the immune response to the virus and the cardiovascular system. In addition, a high prevalence of pre-existing cardio-metabolic disease has been noted among those with severe COVID-19 [16, 17], and those with pre-existing cardiovascular conditions suffer increased mortality during COVID-19 infection [18]. In particular, the reported case fatality rates for COVID-19 are $10.5 \%$ in patients with cardiovascular disease, $7.3 \%$ in patients with diabetes, and $6.0 \%$ in those with hypertension, higher than the case-fatality rate of $3-4 \% \mathrm{ob}-$ served world-wide for patients without these co-morbidities [7••]. Last but not least, the increased frequency of adverse cardiovascular events following the resolution of COVID-19, similar to other viral infections such as influenza [19], may also play a role in worsening the mortality of patients with COVID-19. Thus, understanding the relationship between the viral-host immune response and the cardiovascular system will be critically important in our care and management of patients with COVID-19 going forward.

\section{Biology of SARS-CoV-2}

In order to better understand the biology of viral immune response and how it impacts the heart, we explore here the basic biological mechanisms underlying viral entry into the host cells and the subsequent immune response. Coronaviruses are enveloped viruses with a single-strand, positive-sense RNA genome approximately 26-32 kilobases in size, which is the largest known genome for an RNA virus. Six coronaviruses (CoVs) are known to infect humans: 229E, OC43, SARS-CoV, NL63, HKU1, and MERS-CoV [3]. In humans, $\mathrm{CoV}$ infections primarily involve the upper respiratory tract and GI tract [3]. Studies have demonstrated that SARS-CoV-2, as well as other corona viruses, requires the angiotensin-converting enzyme 2 (ACE2) for cellular entry [20]. ACE2 is a type I integral membrane protein that serves an important role in cardiorenal homeostasis. It is also highly expressed in lung alveolar cells, providing the main entry site for virus into human hosts [21]. It is plausible that the high expression of ACE2 in the lung, gut, heart, and kidneys may facilitate direct damage by the virus throughout the course of infection. One key protein on the virus - the Spike protein (S) - facilitates viral entry into the target cells by the binding of its surface unit, S1, to the ACE2 receptor on the host cell [21-23], followed by cleavage by hostcell protease TMPRSS2 [24]. Other important SARSCoV-2 components include the hemagglutinin-esterase protein, the membrane $(\mathrm{M})$ protein, the nucleocapsid protein, the small envelope protein, the internal protein, and group-specific proteins, which could become targets for vaccines in the future [25]. Of note, SARS-CoV-2 also contains an RNA-dependent RNA polymerase which is the target of the anti-viral agent remdesivir, currently being studied randomized clinical trials for use against COVID-19 disease [26].

\section{The Role of Host Immune Response}

The host immune response to viral entry is also important to discuss, as pathogenesis in the later stages of SARS$\mathrm{CoV}$ and SARS-CoV-2 infection results not only from direct viral toxicity but also from immune dysregulation and hyperactivity [27, 28]. Progress in this field, however, has been hindered by the failure to replicate in mice, ferrats, or non-human primates the lethal human immune response in ARDS with the original SARS-CoV strain $[29,30]$. This has led to the development of mouse- or rat-adapted strains of SARS-CoV that have been able to replicate the extensive and often lethal pulmonary disease [31]. The majority of studies addressing the immune 
response to respiratory viral infections involve mice infected with a variety of natural and mouse-adapted pathogens.

The process of respiratory viral invasion into the body begins with infection of the airway epithelial cells and the activation of lung-resident dentritic cells ( $\mathrm{rDCs}$ ) via acquisition of the invading pathogen or antigens from infected epithelial cells. These rDCs then become activated, process antigen and migrate to the draining (mediastinal and cervical) lymph nodes (DLN). Naïve circulating T cells in the DLNs then recognize antigens presented on the DCs in the form of MHC/peptide complexes. In combination with additional costimatory signals, $\mathrm{T}$ cells then become activated, proliferate, and migrate to the infected site $[32,33]$. Upon arrival to the site of infection, $\mathrm{T}$ cells produce and release antiviral cytokines including interferon (IFN)- $\gamma$, tumor necrosis factor (TNF)- $\alpha$, and interleukin (IL)-2; chemokines including CXC chemokine ligand (CXCL)-9, 10, and, 11, and cytotoxic molecules such as perforin and granzyme B [34]. IFN- $\gamma$ and other effector cytokines directly inhibit viral replication and enhance antigen presentation, while the chemokines released by activated $T$ cells recruit more innate and adaptive cells to combat the pathogen. Granzyme B and other cytotoxic molecules also directly kill infected cells to eliminate the pathogen [35-38].

Recent data from China on SARS-CoV-2 [28, 39] as well as prior data from SARS-CoV [40] demonstrate a rapid reduction of T lymphocytes (both CD4+ and CD8+) in the peripheral blood of infected patients [27, 41, 42]. This is in direct contrast to the proliferative lymphocyte responses seen with other viral infections such as Epstein-Barr Virus (EBV), human immunodeficiency virus (HIV)-1, or cytomegalovirus (CMV), but similar to what happens during other acute viral infections, such as influenza. The loss of lymphocytes precedes even the abnormal radiographic changes on chest $\mathrm{X}$ ray [43•]. Despite reduction in T lymphocyte counts, peripheral blood analysis on a patient infected with SARS-CoV-2 demonstrated increased markers of $\mathrm{T}$ lymphocyte activation [44], as evidenced by high proportions of HLA-DR and CD38 double-positive fractions [28]. Additionally, there was an increased percentage of highly proinflammatory CCR6+ Th17 among CD4+ T cells, and an increased concentration of cytotoxic granules in CD8+ T cells $(31.6 \%$ perforin positive, $64.2 \%$ granulysin positive, and $30.5 \%$ granulysin/perforin double-positive) [28]. Interestingly, one group found that production of IFN- $\gamma$ by CD4+, T cells but not CD8+ T cells or NK cells tended to be lower in severe cases compared with moderate cases [41]. CD4+ T cells, in particular, are felt to be especially important in the host-immune defense against SARS-CoV infections [45]. In addition, disturbances in T regulatory cells (Tregs) were noted in severe cases - with a significantly lower proportion of C45RA+ naïve Tregs (nTregs) and a slightly higher proportion of their memory counterparts
CD45RO+ memory Tregs (mTregs) [41]. On recovery, there is a rapid and significant restoration of $\mathrm{CD} 3+, \mathrm{CD} 4+$, and CD8+ T cells along with $\mathrm{B}$ cell and NK cell counts 23 months after onset of disease [40]. Memory CD4+ T cells returned to normal 1 year after onset, whereas other cell counts including total $\mathrm{T}$ lymphocytes, CD3+, CD4+ and naïve CD4+ T cells were still lower than healthy controls [43॰]. The mechanism of lymphocytopenia in peripheral blood is unclear but thought to be due to sequestration, with release of sequestered cells upon recovery [21]. Taken together, these changes in lymphocyte populations suggest dramatic dysregulation, evidence of T cell "exhaustion" and shifts in the adaptive immune response to SARS-CoV and SARS-CoV-2 infections [42].

In addition to changes in lymphocyte populations, changes in innate immunity likely also contribute to viral pathogenesis, particularly as seen in severe lung and systemic inflammation secondary to cytokine storm. In ARDS, increased levels of cytokines such as TNF- $\alpha$, IFN inducible protein 10 (IP10), IL-6, and IL-8 are thought to contribute to tissue destruction and poor outcomes [46], attributed to hyperactivation of macrophage/monocyte lineage cells. SARS-CoV-2-infected patients have high levels of IL-1 beta, IFN- $\gamma$, IP-10, and monocyte chemoattractant protein-1 (MCP-1), which probably leads to activated T-helper-1 cell response [1]. Compared with patients who did not require ICU admission, those requiring ICU admission had higher conentrations of granulocyte colony-stimulating factors (GCSF), IP-10, MCP-1, macrophage inflammatory protein-1 $\alpha$ (MIP-1- $\alpha$ ), and TNF- $\alpha$, suggesting that cytokine storm might affect disease severity [47]. Additionally, increased levels of type I IFN and a dysregulated IFN-stimulated gene (ISG) response have been seen in patients with severe SARS $[48,49]$.

Last but not least, SARS-specific IgG antibodies are produced in the late acute stage (about 2 weeks from symptom onset), gradually increase throughout the course of the disease and are felt to be associated with disease outcome [50]. The development of anti-SARS-CoV-2 antibodies is highly relevant for both protecting against viral replication/expansion in the infected host as well as providing a source of anti-SARSCoV-2 convalescent plasma to treat patients with severe disease, although supporting data for efficacy is currently lacking. This is being tested in COVID-19 positive patients under an expanded access program by the FDA [51].

\section{Viral Toxicity and Myocardial Injury in COVID-2}

Myocardial injury, manifesting as elevated serum troponin levels, has been described in many patients infected with COVID-19, and mortality has been associated with increase in troponin levels $>99$ th percentile of the upper limit of normal and with electrocardiographic and echocardiographic 
abnormalities [12•, 18, 52]. In addition, reports of the rarer manifestation of fulminant myocarditis with markedly elevated troponin levels have been reported [12•, 13, 14].

There are several thoughts on the mechanism of injury, including direct myocardial injury by the virus through ACE2 entry, hypoxia-induced myocardial injury, microvascular damage and endothelial shedding, and cytokine/ inflammation-mediated damage [15••]. Direct viral toxicity on cardiomyocytes has occurred in the setting of other viral infections such as coxsackievirus-induced myocarditis. In this case, the entry of the coxsackievirus is through the coxsackievirus and adenovirus receptor (CAR) and through release of protease $2 \mathrm{~A}$ coded by coxsackievirus particles, disrupting the dystrophin cytoskeleton complex [53-55]. In the case of coronavirus, as mentioned above, the spike (S) protein of coronaviruses facilitates viral entry into target cells. Entry depends on binding of the surface unit, S1, of the S protein to ACE2, allowing the virus to attach to the surface of the target cell. In addition, entry requires $\mathrm{S}$ protein priming by cellular proteases, which entails $\mathrm{S}$ protein cleavage at the S1/S2 and S2' site and allows fusion of viral and cellular membranes, a process driven by the $\mathrm{S} 2$ subunit [23]. It was found that SARS-2-S shares $76 \%$ amino acid identity with SARS-S, and both engage ACE2 and employ the cellular serine protease TMPRSS2 for S protein priming for host cell entry [22]. Interestingly, injection of SARS-CoV Spike protein into mice worsened acute lung failure in vivo, and was attenuated by blockade of the renin-angiotensin pathway [21]. Also of note, TMPRSS2 is highly expressed in the lung and kidneys, but is present in only low to moderate levels in the heart and blood vessels, suggesting other mechanisms of injury for the latter organ systems [56]. Lastly, the amount of viral load in SARS-CoV-2 infection correlates with disease severity, with higher viral loads on presentation correlating with worse disease outcomes [57]. This study highlights the potential importance of direct viral toxicity in the pathogenesis of COVID-19 infections.

In addition to direct damage caused by the virus, there has also been speculation of an ischemic effect, either in the form of demand ischemia from lung pathology or direct toxicity by the virus on the macro- or microvascular level. It has been suggested that, because ACE2 is expressed on the endothelium, it may induce endothelial shedding and dysfunction contributing to vascular damage, local inflammation, and production of procoagulant factors predisposing to thrombosis, similar to the increase in myocardial infarctions observed after influenza infections $[19,58]$. In addition to endothelial inflammation and dysfunction, an increased incidence of abnormal coagulation parameters and of disseminated intravascular coagulation (DIC) has been noted in patients with SARS-CoV-2 infection [59, $60]$, contributing to risk of thrombosis and ischemic events that could damage the myocardium.
Immune Responses to SARS-CoV-2 Infection and the Heart

Early reports of fulminant myocarditis have alerted the medical and scientific communities that myocardial inflammation may play a role in cardiac injury during viral infection [12•, $13,14]$. However, the exact mechanism of this is currently unclear $[12 \bullet, 13]$, as acute lymphocyte infiltrates were not noted in the myocardium of a SARS-CoV-2-infected ARDS patient autopsy [39], where only a few mononuclear inflammatory cells were seen. Currently, there is great interest in obtaining the pathological specimens from patients presenting with markedly elevated troponin and fulminant myocarditis in order to evaluate for lymphocyte-induced myocardial injury in SARS-CoV-2 infection. Consistent with this, no myocardialspecific epitopes have thus far been identified in the setting of SARS-CoV or SARS-CoV-2 infection. Several HLAA*02:01-specific $\mathrm{T}$ cells recognizing SARS-CoV epitopes have been identified in the peripheral blood mononuclear cells (PBMC) of SARS-recovered individuals, including immunogenic epitopes localized to spike (S) and nucleocapsid (N) protein of SARS-CoV [61, 62]. Of note, MRI-verified acute myocarditis has been reported in association with MERS-CoV [63], although the exact mechanism by which it occurs is unknown.

Although no evidence of direct lymphocytic infiltration of the myocardium exists, the dysregulation of T cells can likely contribute to the cytokine storm and multi-organ damage in the setting of coronavirus infection. A recent retrospective, multi-center study of 150 patients confirmed that inflammatory markers, including elevated ferritin (mean $1297.6 \mathrm{ng} / \mathrm{ml}$ in non-survivors vs $614.0 \mathrm{ng} / \mathrm{ml}$ in survivors, $p<0.001)$ and IL$6(p<0.0001)$ were associated with more severe COVID-19 infection, suggesting that systemic inflammation may be a significant driver of multi-organ damage [18, 64]. A separate group has also reported that the serum cytokines IL-2R, IL-6, IL-10, and TNF- $\alpha$ are increased in patients with severe disease [41]. This systemic release of cytokines, characterized by increased IL-2, IL-6, IL-10, GCSF, IFN- $\gamma$, MCP-1, MIP-1- $\alpha$, and TNF- $\alpha$, likely contributes to cardiac injury in a situation analogous to cardiotoxicity in the setting of chimeric antigen receptor (CAR)-T cell therapy. A prior study demonstrated that cardiac injury and cardiovascular events in the form of elevated troponin and left ventricular systolic dysfunction are common post-CAR-T; in a cohort of 137 patients with postCAR-T cytokine release syndrome (CRS), $21 \%$ had elevated troponin and $12 \%$ developed cardiovascular events including cardiac arrest, decompensated heart failure, and arrhythmias [65]. Notably in this study, a shorter time from CRS onset to the administration of the IL-6 inhibitor tocilizumab was associated with a lower rate of cardiovascular events [65]. Of note, tociluzumab may have some benefit in COVID-19 infection, suggesting a common mechanism of injury in the two settings 
[66]. The exact mechanism by which cytokines/chemokines damage the myocardium is unknown, but cardiomyocyte and endothelial cell death in the presence of inflammatory cytokines such as TNF- $\alpha$ has been well documented in the literature $[67,68]$.

\section{Host-Specific Variations in COVID-19 Immune Response}

Age and gender differences in COVID-19 infection rates have raised interest in possible differences in age- and genderdependent immune responses to viral exposure. Children account for the minority of laboratory-confirmed cases of COVID-19 in China and appear less susceptible to severe disease [69]. Although the functions of both innate and adaptive immune immunity declines with aging, this does not typically start until late adulthood, and thus would not fully explain the decreased severity of disease in children compared with even young adults. [70-72]. The effect of age on the immune system can be demonstrated by the low protective titers among $50 \%$ of adults older than 65 who receive the influenza vaccine [73]. Additional information about the differential response of SARS-CoV and SARS-CoV-2 with aging comes from animal models; in comparison with SARS-CoVMA15-infected young C57BL/6 mice, infection of aged mice (12 months) is associated with severe reduction in the number of virus-specific CD8+ T cells in the lungs [66].

In addition to the effect of aging, gender is also thought to play a role in outcomes in SARS-CoV-2 infection. One study demonstrated a higher incidence of SARS-CoV-2 infection in older adult males compared with females [74]. Sex differences in immune response have been noted in literature, although the reasons are not clear. Males experience greater severity and prevalence of bacterial, viral, fungal and parasitic infections than females, who also mount a more robust response to antigenic challenges including infection and vaccination [75-78]. We (Davis et al) have used machine learning approaches to identify a cluster of genes involved in lipid biosynthesis, previously shown to be upregulated by testosterone, which correlates with poor virus-neutralizing activity in men [79]. Interestingly, the stronger immune response in females is thought to explain why females are more prone to immunemediated pathologies including autoimmune disease and cytokine storm [78]. However, in the case of COVID-19, there is no data to currently support a female-predominance toward cytokine storm; if anything, males are prone to more severe disease and mortality [74]. Thus, it would be important to gather more data and larger number cohort studies on the current SARS-CoV-2 pandemic to study further delineate whether there is a gender-dependent risk for cytokine storm and subsequent cardiac injury in COVID-19 infection.

\section{Treatments for SARS-CoV-2 Infection}

Various therapies have emerged to treat the various aspects of viral pathogenesis and subsequent immune response, and an understanding of which aspect of disease pathogenesis they target may aide the clinician in knowing when to use them. Treatments which target steps early in the infection process ("respiratory phase" in Fig. 1) are meant to suppress viral replication and aid the host immune response to fight the virus. When the immune response becomes hyperactive ("sepsis/shock" phase in Fig. 1) with cytokine storm, immunomodulators that target various harmful inflammatory cytokines may help mitigate end-organ toxicity. Unfortunately, there are currently no FDA-approved therapies for SARS-CoV-2, and no substantial randomized trial data to support any therapy thus far. However, several promising therapies are actively being test in patients including the recently announced multicenter, randomized SOLIDARITY trial (remdesivir, hydroxychloroquine) sponsored by the World Health Organization [51].

Anti-microbial agents which target the early (pre-systemic) phase of infection include remdesivir, hydroxychloroquine, and azithromycin. Remdesivir is a drug which targets the RNA polymerase, suppresses viral replication, and has strong in vitro evidence of efficacy against SARS-CoV-2 [26]. Although randomized trial data are still pending [51], there have been anecdotal reports of improvement after compassionate use of remdesivir [80]. Lopinavir-ritonavir is another protease inhibitor which showed initial promise, but a recently published study from Beijing showed no significant difference in the treatment versus control group, suggesting that the effect, if present, was insufficient to cause a significant clinical response [81]. Chloroquine has traditionally been used as an anti-malaria drug, but has shown reasonable efficacy against SARS-CoV-2 in vitro [26]. A single arm study of 20 COVID-19-positive patients treated in France demonstrated some efficacy for the combination of azithromycin and hydroxychloroquine, a safer variation of chloroquine, in SARS-CoV-2 [80]. Another small non-randomized study showed similar results [82] However, these were small, non-randomized studies in whose definition of efficacy was based on viral clearance rather than mortality benefit. A subsequent pilot randomized trial in Shanghai comparing hydroxychloroquine and placebo has shown no difference in virologic clearance [83]. Azithromycin is an antimicrobial agent generally used for antibacterial purposes; however, it also has been shown to inhibit zika virus tropism in the human brain and has been suggested as an adjunct for treatment of intracellular microbes in the past $[80,81]$. Since both chloroquine/ hydroxychloroquine and azithromycin lead to corrected QT (QTC) prolongation, their use either alone or in combination should be monitored for this potential cardiotoxic side effect [84].

The second group of therapies is immunomodulators, used to target immune hyperactivity and the cytokine storm that 


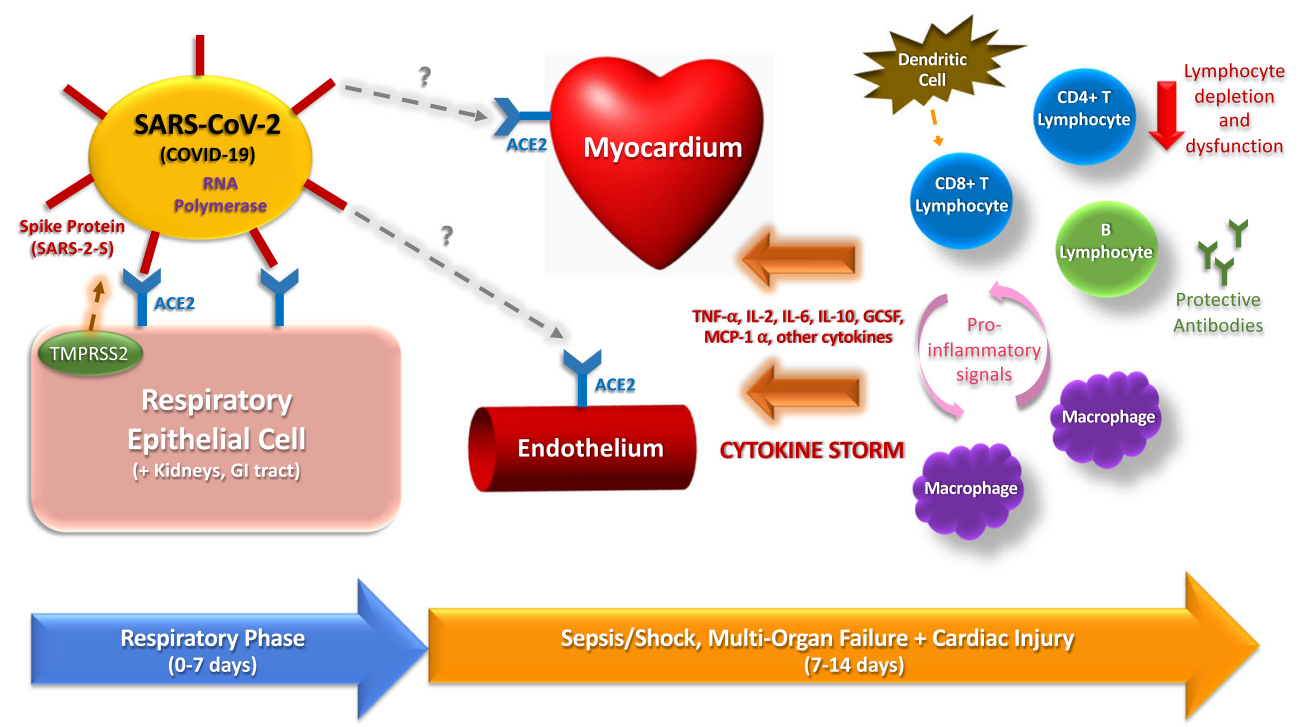

Fig. 1 Hypothesis of SARS-CoV-2 pathogenesis and immune response in cardiovascular injury. Spike protein (SARS-2-S) on the virus is activated by cellular serine protease TMPRSS2 highly expressed in lung, renal, and gastrointestinal cells and engages with angiotensinconverting enzyme 2 (ACE2) that is highly expressed on respiratory epithelial cells for entry into the host cell. Early infection is

occurs in the later stages of COVID-19 infection [64]. Monoclonal antibodies have shown some promise in early studies. The IL-6 inhibitors tocilizumab and sarilumab have shown early benefit and are being studied in ongoing randomized trials [51]. Other therapies including meplazumab (antiCD147), eculizumab (targets complement), adalimumab (TNF- $\alpha$ inhibitor), and IVIG (saturation of Fc receptors on macrophages, suppression of chemokines/cytokines) are also intended toward reduction of deleterious immune effects [51]. Interestingly, immune stimulatory agents aimed at enhancing beneficial aspects of the immune response are also being tested - including IFN- $\beta 1 \mathrm{a}$, PD-1 inhibitors, and donor convalescent plasma [51]. There are conflicting data to support the use of glucocorticoids in COVID-19 related ARDS [85], and even potential evidence of harm in the form of decreased viral clearance in SARS and MERS [86] and increased mortality in SARS-CoV2 [87]. Without evidence of acute lymphocytic myocarditis in cardiovascular injury, we do not currently advocate for the routine administration of glucocorticoids for elevated troponin in COVID-19 patients. Randomized trials on both glucocorticoids and IVIG in severe cases of COVID-19 are ongoing [51].

\section{Conclusion}

SARS-CoV-2 has become a worldwide health threat, with the numbers of infected patients growing rapidly. An increased incidence of cardiac injury has been observed among those with severe infection. The mechanism of cardiac injury is characterized by viral replication and direct damage by the virus to host cells, via ACE2/TMPRSS2-mediated cell entry. As infection progresses, pro-inflammatory signals upregulate inflammatory cytokine production by cells in the adaptive and innate immune system, leading to cytokine storm and multi-organ damage

unclear but likely involves a combination of direct viral damage and immune-mediated damage by inflammatory cytokines/chemokines and cytotoxic immune cell response in the later stages of infection. The host immune response and contributors to cytokine storm in SARS-CoV-2 infection are complex. Significant depletion and dysregulation of $\mathrm{T}$ lymphocytes may contribute to immune dysregulation and hyperactivity. Cardiac damage in the setting of cytokine storm may be analogous to that seen in cardiotoxicity from CAR-T. Treatments for COVID-19 are bimodal, with one group of treatments targeted toward early infection and viral replication, and another group targeted toward immune modulation in the later, systemic inflammatory phase of infection. With the advent of single cell immune phenotyping technologies, it will be important to perform comprehensive immune surveys of infected patients to better understand systemic perturbations with the infection and downstream cardiovascular effects. More data are needed to help guide us toward definitive treatment and protection of the cardiovascular system in COVID-19 infection.

\section{Compliance with Ethical Standards}

Conflict of Interest Han Zhu, June Rhee, Paul Cheng, Sarah Waliany, Amy Chang, Ronald M. Witteles, Holden Maecker, Mark M. Davis, Patricia K. Nguyen, and Sean M. Wu declare that they have no conflict of interest.

Human and Animal Rights and Informed Consent This article does not contain any studies with human or animal subjects performed by any of the authors. 


\section{References}

Papers of particular interest, published recently, have been highlighted as:

- Of importance

-• Of major importance

1. Huang C, Wang Y, Li X, Ren L, Zhao J, Hu Y, et al. Clinical features of patients infected with 2019 novel coronavirus in Wuhan, China. Lancet. 2020;395(10223):497-506.

2. Wang C, Horby PW, Hayden FG, Gao GF. A novel coronavirus outbreak of global health concern. Lancet. 2020;395(10223):470 3.

3. Su S, Wong G, Shi W, Liu J, Lai ACK, Zhou J, et al. Epidemiology, genetic recombination, and pathogenesis of coronaviruses. Trends Microbiol. 2016;24(6):490-502.

4. Wang D, Hu B, Hu C, Zhu F, Liu X, Zhang J, et al. Clinical characteristics of 138 hospitalized patients with 2019 novel coronavirus-infected pneumonia in Wuhan, China. JAMA - J Am Med Assoc. 2020;323(11):1061-9.

5. Zhou F, Yu T, Du R, Fan G, Liu Y, Liu Z, et al. Articles Clinical course and risk factors for mortality of adult inpatients with COVID-19 in Wuhan, China: a retrospective cohort study. Lancet. 2020;6736(20):1-9.

6. Xu Z, Li S, Tian S, Li H, Kong L. Full spectrum of COVID-19 severity still being depicted. Lancet. 2020;395(10228):947-8.

7.• Wu Z, McGoogan JM. Characteristics of and important lessons from the coronavirus disease 2019 (COVID-19) outbreak in China: summary of a report of 72314 cases from the Chinese Center for Disease Control and Prevention. JAMA. 2020;2019:36 This article is of major importance because it demonstrates increased case falality rates in COVID-19 patients with concommitant cardiovascular co-morbidities in a large cohort.

8. Dong E, Du H, Gardner L. An interactive web-based dashboard to track COVID-19 in real time. Lancet Infect Dis. 2020;3099(20):19 20 .

9. Zhang S, Diao MY, Yu W, Pei L, Lin Z, Chen D. Estimation of the reproductive number of novel coronavirus (COVID-19) and the probable outbreak size on the diamond princess cruise ship: a data-driven analysis. Int J Infect Dis. 2020;93:201-4.

10. Driggin E, Madhavan M V., Bikdeli B, Chuich T, Laracy J, BondiZoccai G, et al. Cardiovascular considerations for patients, health care workers, and health systems during the coronavirus disease 2019 (COVID-19) pandemic. J Am Coll Cardiol. 2020.

11. Arentz M, Yim E, Klaff L, Lokhandwala S, Riedo FX, Chong M, et al. Characteristics and outcomes of 21 critically ill patients with COVID-19 in Washington state. JAMA. 2020.

12. Hu H, Ma F, Wei X, Fang Y. Coronavirus fulminant myocarditis saved with glucocorticoid and human immunoglobulin Hongde. Eur Heart J. 2020;1307800. This paper is of importance because it describes an example of fulminant myocarditis which can occur in association with COVID-19 infection.

13. Chen C, Zhou Y, Wang DW. SARS-CoV-2: a potential novel etiology of fulminant myocarditis. Herz. 2020;10-2.

14. Zeng JH, Liu Y-X, Yuan J, Wang F-X, Wu W-B, Li J-X, et al. First case of COVID-19 infection with fulminant myocarditis complication: case report and insights. 2020

15.• Shi S, Qin M, Shen B, Cai Y, Liu T, Yang F, et al. Association of Cardiac Injury with mortality in hospitalized patients with COVID19 in Wuhan, China. JAMA Cardiol. 2020;1-8. This paper is of major importance because it is the first large retrospective study demonstrating cardiac injury as an independent predictor of mortality in COVID-19 infection.
16. Zheng Y-Y, Ma Y-T, Zhang J-Y, Xie X. COVID-19 and the cardiovascular system. Nat Rev Cardiol. 2020

17. Liu Y, Li J, Liu D, Song H, Chen C, Lv M, et al. Clinical features and outcomes of 2019 novel coronavirus-infected patients with 1 cardiac injury.

18. Ruan Q, Yang K, Wang W, Jiang L, Song J. Clinical predictors of mortality due to COVID-19 based on an analysis of data of 150 patients from Wuhan, China. Intensive Care Med. 2020.

19. Waxman DA, Kanzaria HK, Schriger DL. Acute myocardial infarction after laboratory-confirmed influenza infection. N Engl J Med. 2018;378(26):2538-41.

20. Ge X, Li J, Yang X, Chmura AA, Zhu G, Epstein H, et al. Isolation and characterization of a bat SARS-like coronavirus that uses the ACE2 receptor parts of the experiments and analyses HHS public access. Z Conduct Nat. 2013;503(7477):535-8.

21. Kuba K, Imai Y, Rao S, Gao H, Guo F, Guan B, et al. A crucial role of angiotensin converting enzyme 2 (ACE2) in SARS coronavirusinduced lung injury. Nat Med. 2005;11(8):875-9.

22. Taylor RL. Structure of SARS coronavirus spike receptor-binding domain Complexed with receptor. J Clin Endocrinol Metab. 1950;10(10):1361-2.

23. Hoffmann M, Kleine-Weber H, Schroeder S, Mü MA, Drosten C, Pö S, et al. SARS-CoV-2 cell entry depends on ACE2 and TMPRSS 2 and is blocked by a clinically proven protease inhibitor article SARS-CoV-2 cell entry depends on ACE2 and TMPRSS2 and is blocked by a clinically proven protease inhibitor. Cell. 2020 .

24. Matsuyama S, Nagata N, Shirato K, Kawase M, Takeda M, Taguchi F. Efficient activation of the severe acute respiratory syndrome coronavirus spike protein by the transmembrane protease TMPRSS2. J Virol. 2010 Dec 15;84(24):12658-64.

25. Weiss SR, Navas-Martin S. Coronavirus pathogenesis and the emerging pathogen severe acute respiratory syndrome coronavirus. Microbiol Mol Biol Rev. 2005;69(4):635-64.

26. Wang M, Cao R, Zhang L, Yang X, Liu J, Xu M, et al. Remdesivir and chloroquine effectively inhibit the recently emerged novel coronavirus (2019-nCoV) in vitro. Cell Research. Springer Nature; 2020.

27. Qin C, Zhou L, et al. Dysregulation of immune response in patients with COVID-19 in Wuhan. China Chuan J Chem Inf Model. 2013;53(9):1689-99.

28. Xu Z, Shi L, Wang Y, Zhang J, Huang L, Zhang C, et al. Pathological findings of COVID-19 associated with acute respiratory distress syndrome. Lancet Respir Med. 2020;2600(20):19-21.

29. Subbarao K, Roberts A. Is there an ideal animal model for SARS? Trends Microbiol. 2006;14(7):299-303.

30. van den Brand JMA, Haagmans BL, van Riel D, Osterhaus ADME, Kuiken T. The pathology and pathogenesis of experimental severe acute respiratory syndrome and influenza in animal models. J Comp Path. 2014;151:83-112.

31. Day CW, Baric R, Cai SX, Frieman M, Kumaki Y, Morrey JD, et al. A new mouse-adapted strain of SARS-CoV as a lethal model for evaluating antiviral agents in vitro and in vivo. Virology. 2009;395(2):210-22.

32. Larsson M, Messmer D, Somersan S, Fonteneau J-F, Donahoe SM, Lee M, et al. Requirement of mature dendritic cells for efficient activation of influenza A-specific memory CD8 + T cells. J Immunol. 2000;165(3):1182-90.

33. Belz GT, Smith CM, Kleinert L, Reading P, Brooks A, Shortman K, et al. Distinct migrating and nonmigrating dendritic cell population are involved in MHC class I-restricted antigen presentation after lung infection with virus. Proc Natl Acad Sci U S A. 2004;101(23):8670-5.

34. Channappanavar R, Zhao J, Perlman S. T cell-mediated immune response to respiratory coronaviruses. Immunol Res. 2014;59(1-3): $118-28$. 
35. Cerwenka A, Morgan TM, Dutton RW. Naive, effector, and memory CD8 T cells in protection against pulmonary influenza virus infection: homing properties rather than initial frequencies are crucial. J Immunol. 1999;163(10):5535-43.

36. Cerwenka A, Morgan TM, Harmsen AG, Dutton RW. Migration kinetics and final destination of type 1 and type $2 \mathrm{CD} 8$ effector cells predict protection against pulmonary virus infection. J Exp Med. 1999;189(2):423-34.

37. Saha B, Jyothi Prasanna S, Chandrasekar B, Nandi D. Gene modulation and immunoregulatory roles of interferon $\gamma$. Cytokine. 2010;50(1):1-14.

38. Román E, Miller E, Harmsen A, Wiley J, Von Andrian UH, Huston $\mathrm{G}$, et al. CD4 effector T cell subsets in the response to influenza: heterogeneity, migration, and function. J Exp Med. 2002;196(7): 957-68.

39. Luo W, Hong Y, Gou J, Li X, Sun Y, Li J, et al. Clinical pathology of critical patient with coronavirus pneumonia (COVID-19). PrePrints. 2020;1-14.

40. Li T, Qiu Z, Zhang L, Han Y, He W, Liu Z, et al. Significant changes of peripheral $\mathrm{T}$ lymphocyte subsets in patients with severe acute respiratory syndrome. J Infect Dis. 2004;189(4):648-51.

41. Chen G, Wu D, Guo W, Cao Y, Huang D, Wang H, et al. Clinical and immunologic features in severe and moderate forms of coronavirus disease 2019. medRxiv. 2020;2020.02.16.20023903.

42. Diao B, Wang C, Tan Y, Chen X, Liu Y, Ning L, et al. Reduction and functional exhaustion of $\mathrm{T}$ cells in patients with coronavirus disease 2019 (COVID-19). medRxiv. 2020;2019:1-14.

43. Lin L, Lu L, Cao W, Li T. Hypothesis for potential pathogenesis of SARS-CoV-2 infection - a review of immune changes in patients with viral pneumonia. Emerg Microbes Infect. 2020;0(0):1-14 This paper is of importance because it describes changes in immune cell subsets in patients with COVID-19 throughout the course of infection.

44. Thevarajan I, Nguyen THO, Koutsakos M, Druce J, Caly L, van de Sandt CE, et al. Breadth of concomitant immune responses prior to patient recovery: a case report of non-severe COVID-19. Nat Med. $2020 ; 1$.

45. Chen J, Lau YF, Lamirande EW, Paddock CD, Bartlett JH, Zaki SR, et al. Cellular immune responses to severe acute respiratory syndrome coronavirus (SARS-CoV) infection in senescent BALB/c mice: CD4+ T cells are important in control of SARS-CoV infection. J Virol. 2010;84(3):1289-301.

46. Kong SL, Chui P, Lim B, Salto-Tellez M. Elucidating the molecular physiopathology of acute respiratory distress syndrome in severe acute respiratory syndrome patients. Virus Res. 2009;145(2):260 9.

47. Fong PC, Boss DS, Yap TA, Tutt A, Wu P, Mergui-Roelvink M, et al. A novel coronavirus associated with severe acute respiratory syndrome. N Engl J Med. 2009;361(2):123-34.

48. Baas T, Taubenberger J, Chong P, Chui P, Katze M. SARS-CoV virus-host interactions and comparative etiologies of acute respiratory distress syndrome as determined by transcriptional and cytokine profiling of formalin-fixed paraffin-embedded tissues. J Interf Cytokines Res. 2006;26(5):309-17.

49. Wong CK, Lam CWK, Wu AKL, Ip WK, Lee NLS, Chan IHS, et al. Plasma inflammatory cytokines and chemokines in severe acute respiratory syndrome. Clin Exp Immunol. 2004;136(1):95103.

50. Cao Z, Liu L, Du L, Zhang C, Jiang S, Li T, et al. Potent and persistent antibody responses against the receptor-binding domain of SARS-CoV spike protein in recovered patients. Virol J. 2010;7(1):299.

51. Overview of planned or ongoing studies of drugs for the treatment of COVID-19. IEICE ESS Fundam Rev. 2020;13(3):177.

52. Yang X, Yu Y, Xu J, Shu H, Xia J, Liu H, et al. Clinical course and outcomes of critically ill patients with SARS-CoV-2 pneumonia in
Wuhan, China: a single-centered, retrospective, observational study. Lancet Respir Med. 2020;2600(20):1-7.

53. Herman DS, Lam L, Taylor MRG, Wang L, Teekakirikul P, Christodoulou D, et al. Interaction of coxsackievirus B3 with the full length coxsackievirus-adenovirus receptor. Nat Struct Biol. 2001;8(10):874-8.

54. Zanatta A, Carturan E, Rizzo S, Basso C, Thiene G. Story telling of myocarditis. Int J Cardiol. 2019;294:61-4.

55. Razzano D, Fallon JT. Myocarditis: somethings old and something new. Cardiovasc Pathol. 2020;44.

56. Vaarala MH, Porvari KS, Kellokumpu S, Kyllönen AP, Vihko PT. Expression of transmembrane serine protease TMPRSS2 in mouse and human tissues. J Pathol. 2001;193(1):134-40.

57. Liu Y, Yan L-M, Wan L, Xiang T-X, Le A, Liu J-M, et al. Viral dynamics in mild and severe cases of COVID-19. Lancet Infect Dis. 2019;2020(20):2019-20.

58. Smeeth L, Thomas SL, Hall AJ, Hubbard R, Farrington P, Vallance P. Risk of myocardial infarction and stroke after acute infection or vaccination. N Engl J Med. 2004;351(25):2611-8.

59. Driggin E, Madhavan M V, Bikdeli B, Laracy J, Bondi-zoccai G, Tyler S, et al. Cardiovascular considerations for patients, health care workers, and health systems during the coronavirus disease 2019 (COVID-19) pandemic. J Am Coll Cardiol. 2020.

60. Tang N, Li D, Wang X, Sun Z. Abnormal coagulation parameters are associated with poor prognosis in patients with novel coronavirus pneumonia. J Thromb Haemost. 2020;(February):1-4.

61. Xu X, Gao X. Immunological responses against SARS-coronavirus infection in humans. Cell Mol Immunol. 2004;1(2):119-22.

62. Wang Y-D, Sin W-YF XG-B, Yang H-H, Wong T-Y, Pang X-W, et al. T-cell epitopes in severe acute respiratory syndrome (SARS) coronavirus spike protein elicit a specific $\mathrm{T}$-cell immune response in patients who recover from SARS. J Virol. 2004;78(14):7861.

63. Alhogbani T. Acute myocarditis associated with novel Middle East respiratory syndrome coronavirus. Ann Saudi Med. 2016;36(1):7880.

64. Mehta P, Mcauley DF, Brown M, Sanchez E, Tattersall RS, Manson JJ, et al. Correspondence COVID-19: consider cytokine storm syndromes and immunosuppression. Lancet. 2020;6736(20):19-20.

65. Alvi RM, Frigault MJ, Fradley MG, Jain MD, Mahmood SS, Awadalla M, et al. Cardiovascular events among adults treated with chimeric antigen receptor T-cells (CAR-T). J Am Coll Cardiol. 2019;74(25):3099-108

66. Xu X, Han M, Li T, Sun W, Wang D, Fu B, et al. Effective treatment of severe COVID-19 patients with Tocilizumab. 2020;

67. Clyne AM, Zhu H, Edelman ER. Elevated fibroblast growth factor2 increases tumor necrosis factor- $\alpha$ induced endothelial cell death in high glucose. J Cell Physiol. 2008 Oct;217(1):86-92.

68. Jarrah AA, Schwarskopf M, Wang ER, LaRocca T, Dhume A, Zhang S, et al. SDF-1 induces TNF-mediated apoptosis in cardiac myocytes. Apoptosis. 2018;23(1):79-91.

69. Lee PI, Hu YL, Chen PY, Huang YC, Hsueh PR. Are children less susceptible to COVID-19? J Microbiol Immunol Infect. 2020; (xxxx):19-20.

70. Zhao J, Zhao J, Legge K, Perlman S. Age-related increases in PGD 2 expression impair respiratory DC migration, resulting in diminished $\mathrm{T}$ cell responses upon respiratory virus infection in mice. $\mathrm{J}$ Clin Invest. 2011;121(12):4921-30.

71. De Bourcy CFA, Lopez Angel CJ, Vollmers C, Dekker CL, Davis MM, Quake SR. Phylogenetic analysis of the human antibody repertoire reveals quantitative signatures of immune senescence and aging. Proc Natl Acad Sci U S A. 2017;114(5):1105-10.

72. Wang C, Liu Y, Xu LT, Jackson KJL, Roskin KM, Pham TD, et al. Effects of aging, Cytomegalovirus infection, and EBV infection on human B cell repertoires. J Immunol. 2014;192(2):603-11.

73. Govaert TM, Thijs CM, Masurel N, Sprenger MJW, Dinant GJ, Knottnerus JA. The efficacy of influenza vaccination in elderly 
individuals. A randomized double-blind placebo-controlled trial. JAMA - J Am Med Assoc. 1994;272(21):1661-5.

74. Chen N, Zhou M, Dong X, Qu J, Gong F, Han Y, et al. Epidemiological and clinical characteristics of 99 cases of 2019 novel coronavirus pneumonia in Wuhan, China: a descriptive study. Lancet. 2020;395(10223):507-13.

75. Klein SL, Poland GA. Personalized vaccinology: one size and dose might not fit both sexes. Vaccine. 2013;31(23):2599-600.

76. Klein SL. The effects of hormones on sex differences in infection: from genes to behavior. Neurosci Biobehav Rev. 2000;24(6):62738 .

77. Di Florio DN, Sin J, Coronado MJ, Atwal PS, Fairweather D. Sex differences in inflammation, redox biology, mitochondria and autoimmunity. Redox Biol. 2020;(September 2019):101482.

78. Robinson DP, Lorenzo ME, Jian W, Klein SL. Elevated 17 $\beta$ estradiol protects females from influenza a virus pathogenesis by suppressing inflammatory responses. PLoS Pathog. 2011;7(7).

79. Furman D, Hejblum BP, Simon N, Jojic V, Dekker CL, Thiebaut R, et al. Systems analysis of sex differences reveals an immunosuppressive role for testosterone in the response to influenza vaccination. Proc Natl Acad Sci U S A. 2014;111(2):869-74.

80. Wang M, Cao R, Zhang L, Yang X, Liu J, Xu M, et al. Remdesivir and chloroquine effectively inhibit the recently emerged novel coronavirus (2019-nCoV) in vitro. Cell Res. 2020;30(3):269-71.

81. Cao B, Wang Y, Wen D, Liu W, Wang J, Fan G, et al. A trial of Lopinavir-ritonavir in adults hospitalized with severe COVID-19. N Engl J Med. 2020.
82. Gautret P, Lagier J-C, Parola P, Hoang VT, Meddeb L, Mailhe M, et al. Hydroxychloroquine and azithromycin as a treatment of COVID-19: results of an open-label non-randomized clinical trial. Int J Antimicrob Agents. 2020;105949.

83. Chen J, Liu D, Liu P, Xu Q, Xia L, Ling Y, et al. A pilot study of hydroxychloroquine in treatment of patients with common coronavirus disease-19 (COVID-19). Zhejiang Univ Med Sci. 2020;(March):3-8.

84. Hancox JC, Vieweg WVR, Hasnain M, Crouse ELB, Baranchuk A. Azithromycin, cardiovascular risks, QTc interval prolongation, torsade de pointes, and regulatory issues: a narrative review based on the study of case reports. Ther Adv Infect Dis. 2013;1(5):155-65.

85. Wu C, Chen X, Cai Y, Xia J, Zhou X, Xu S, et al. Risk factors associated with acute respiratory distress syndrome and death in patients with coronavirus disease 2019 pneumonia in Wuhan, China, JAMA Intern Med 2020;1-10.

86. Hui DS. Systemic corticosteroid therapy may delay viral clearance in patients with middle east respiratory syndrome coronavirus infection. Am J Respir Crit Care Med. 2018;197(6):700-1.

87. Wang Z, Yang B, Li Q, Wen L, Zhang R. Clinical features of 69 cases with coronavirus disease 2019 in Wuhan, China. Clin Infect Dis. 2020.

Publisher's Note Springer Nature remains neutral with regard to jurisdictional claims in published maps and institutional affiliations. 